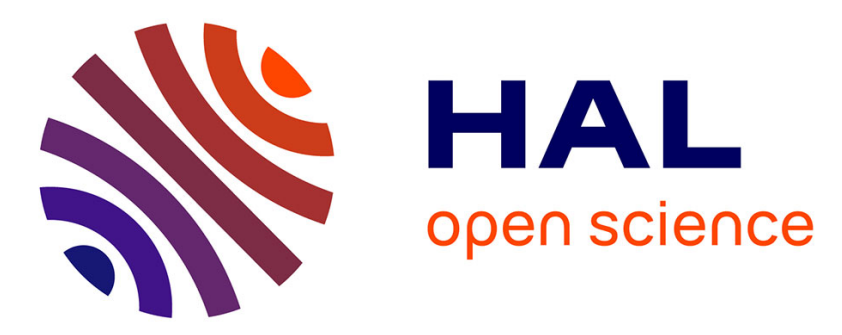

\title{
Characterizing piezoscanner hysteresis and creep using optical levers and a reference nanopositioning stage.
}

\author{
H. Xie, M. Rakotondrabe, Stéphane Régnier
}

\section{To cite this version:}

H. Xie, M. Rakotondrabe, Stéphane Régnier. Characterizing piezoscanner hysteresis and creep using optical levers and a reference nanopositioning stage.. Review of Scientific Instruments, 2009, 80 (4), pp.046102-1/046102-3. 10.1063/1.3115184 . hal-00404732

\section{HAL Id: hal-00404732 \\ https://hal.science/hal-00404732}

Submitted on 17 Jul 2009

HAL is a multi-disciplinary open access archive for the deposit and dissemination of scientific research documents, whether they are published or not. The documents may come from teaching and research institutions in France or abroad, or from public or private research centers.
L'archive ouverte pluridisciplinaire HAL, est destinée au dépôt et à la diffusion de documents scientifiques de niveau recherche, publiés ou non, émanant des établissements d'enseignement et de recherche français ou étrangers, des laboratoires publics ou privés. 


\title{
Characterizing piezoscanner hysteresis and creep using optical levers and a reference nanopositioning stage
}

\author{
H. Xie, ${ }^{1}$ M. Rakotondrabe, ${ }^{2}$ and S. Régnier ${ }^{1}$ \\ ${ }^{1}$ Institute of Intelligent System and Robotics, University of Pierre and Marie Curie/CNRS UMR 7222, \\ 4 Place Jussieu, 75005 Paris, France \\ ${ }^{2}$ Department of Automatic Control and Micro-Mechatronic Systems, FEMTO-ST Institute, \\ CNRS UMR 6174-UFC/ENSMM/UTBM, 24, Rue Alain Savary, 25000 Besançon, France
}

(Received 21 January 2009; accepted 15 March 2009; published online 6 April 2009)

\begin{abstract}
A method using atomic force microscope (AFM) optical levers and a reference nanopositioning stage has been developed to characterize piezoscanner hysteresis and creep. The piezoscanner is fixed on a closed-loop nanopositioning stage, both of which have the same arrangement on each axis of the three spatial directions inside the AFM-based nanomanipulation system. In order to achieve characterization, the optical lever is used as a displacement sensor to measure the relative movement between the nanopositioning stage and the piezoscanner by lateral tracking a well-defined slope with the tapping mode of the AFM cantilever. This setup can be used to estimate a piezoscanner's voltage input with a reference displacement from the nanopositioning stage. The hysteresis and creep were accurately calibrated by the method presented, which use the current setup of the AFM-based nanomanipulation system without any modification or additional devices.
\end{abstract}

Positioning precision is one of the most critical issues in atomic force microscope (AFM) applications. Among several factors that cause positioning errors in the AFM, the most significant are piezoscanner hysteresis and creep. Charge sources were initially proposed to eliminate hysteresis and the creep of the piezoscanner. ${ }^{1,2}$ However, until now, the charge source has not been widely applied due to difficulties in driving such a huge capacitor with commercially available amplifiers. Another method to minimize hysteresis is to model the nonlinear behavior of the piezoscanner. A number of models have been proposed to characterize and compensate for hysteresis, such as the Jiles-Atherton model, ${ }^{3}$ the Preisach model, ${ }^{4,5}$ the Domain Wall Model, ${ }^{6}$ the Maxwell model, ${ }^{7}$ and the Prandtl-Ishlinskii (PI) operator. ${ }^{8-10}$ The PI operator is widely used in real-time applications due to its accuracy and its simplicity of implementation. A logarithmic function is used to compensate for creep by establishing an opposite logarithmic model for the applied voltage so that the final piezoelectric strain will remain constant. ${ }^{11}$ Another approach is to model the creep using a linear dynamic operator. ${ }^{12}$ When characterizing hysteresis and creep, various displacement sensors, including capacitive sensors, inductive sensors, and laser sensors, have generally been used to measure the displacement of the piezoscanner. Recently, the scanning probe microscopy (SPM) itself has been used as its own lateral displacement sensor to determine the compensator parameters. ${ }^{10}$

In our two-tip AFM-based nanomanipulation system, two positioning devices, including a closed-loop nanopositioning stage and an open-loop piezoscanner, are used for motion coordination between two collaborative AFM tips. In this case, both of the positioning devices should have the same displacement scale to achieve prompt and accurate positioning between the AFM tips and samples. We are therefore presenting here a new method for the piezoscanner characterization. In this method, an AFM optical lever, which has been accurately calibrated before application, ${ }^{13,14}$ is used as a lateral displacement sensor to measure displacement of the piezoscanner. By this means, the voltage input of the piezoscanner can be accurately estimated from the reference displacement. The hysteresis and creep are clearly identified using the current setup of the AFM-based nanomanipulation system without any modification or additional equipments.

The system configuration is shown in Fig. 1, in which the piezoscanner (PI P-153.10H) is fixed on the nanopositioning stage (MCL Nano-Bio2M) with the same axis arrangement on all the three spatial directions. In this setup, the AFM optical lever (on tip I) is used as a displacement sensor to measure the relative movement between the nanopositioning stage and the piezoscanner by lateral tracking well-defined slopes (AFM calibration grating TGF11 with a slope angle of $54^{\circ} 44^{\prime}$ ). In order to avoid tip-slope friction effects on tracking accuracy, AFM cantilever (NCLR, Nanoworld) tapping mode is used rather than contact mode. For $X$-axis and the $Y$-axis calibration, the grating is aligned in such a way that grating ridges are perpendicular to the lateral tracking direction. On the other hand, tracking the grating flat surface instead of the sloped surface can be used to calibrate the $Z$-axis. This method can be very accurate because AFM can image features from the nanoscale to the atomic scale. Thanks to the high resolution of the nanopositioning stage $(0.1 \mathrm{~nm}$ with resonant frequency of $300-500 \mathrm{~Hz})$ and the excellent dynamic performance of the piezoscanner (about $1 \mathrm{kHz}$ on $1 \mu \mathrm{m}$ motion amplitude using the amplifier PI E-413 with $\pm 5 \mathrm{~V}$ input and $\pm 250 \mathrm{~V}$ output), the piezoelectric is used to track the motion of the nanopositioning stage that provides a reference displacement. During the tracking, the feedback, that is the voltage output of the photodiode $\Delta V_{\mathrm{PSD}}$, presents displacement errors on the $Z$-axis caused by the relative displacement $d_{e}$ between the nanopositioning stage and the piezoscanner on each axis. $d_{e}$ can be estimated as 


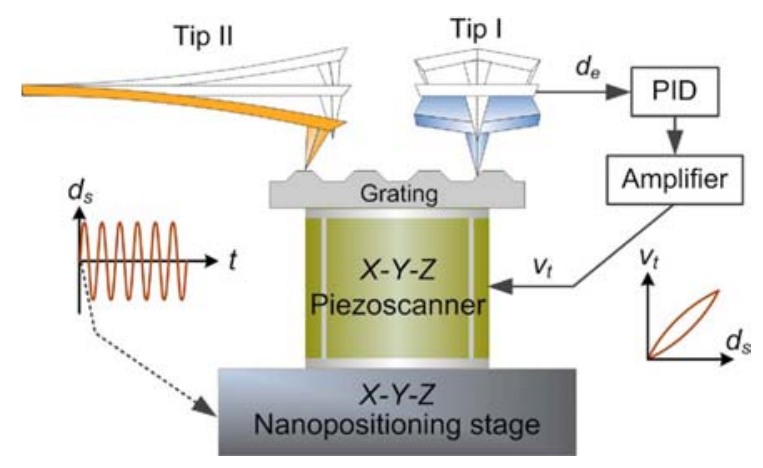

FIG. 1. (Color online) Configuration of the two-tip AFM nanomanipulation system and the calibration setup of the piezoscanner. For calibration, the relative movement between the piezoscanner and the nanopositioning stage is detected using the tip I by tracking a well-defined slope (TGF11) with the tapping mode of the cantilever. An amplifier is used to generate a voltage input $v_{t}$ for the piezoscanner with a reference displacement $d_{s}$ from the nanopositioning stage.

$$
\left\{\begin{array}{l}
d_{e}=\frac{\Delta V_{\mathrm{PSD}} \times S_{\text {tapping }}}{\tan 54^{\circ} 44^{\prime}} \text { on the } X \text {-, } Y \text {-axis } \\
d_{e}=\Delta V_{\mathrm{PSD}} \times S_{\text {tapping }}
\end{array},\right.
$$

where $S_{\text {tapping }}$ is the sensitivity of the optical lever with the cantilever tapping mode. In our system, $S_{\text {tapping }}$ was calibrated as 0.143 and $0.151 \mathrm{~nm} / \mathrm{mV}$ (amplitude) with the cantilever I and the cantilever II, respectively. As shown in Fig. 1 , after PID modulation, an amplifier is used to generate a voltage input $v_{t}$ for the piezoscanner with a reference displacement $d_{s}$ from the nanopositioning stage. To characterize and then compensate for the hysteresis of the piezoscanner, the following procedure is proposed:

(1) A sinusoidal input (as per the left schematic inset in Fig. 1 ) is applied on each axis of the nanopositioning stage with a frequency of $0.5 \mathrm{~Hz}$ and with a $p$ - $p$ displacement of $10 \mu \mathrm{m}$ (Z-axis) or $12 \mu \mathrm{m}(X$-, $Y$-axis $) . B y$ tracking the slope with the dithering AFM cantilever, it is possible to plot a hysteresis behavior from the input voltage $v_{t}$ versus the displacement $d_{s}$ on each axis of the piezoscanner (as per the right schematic inset in Fig. 1).

(2) Inverse the plot of $v_{t}$ versus $d_{s}$ to $d_{s}$ versus $v_{t}$ and use the corresponding hysteresis curve. Then identify parameters of the PI hysteresis model using the inversed curve [Fig. 2(a)]. The identified PI hysteresis model will serve

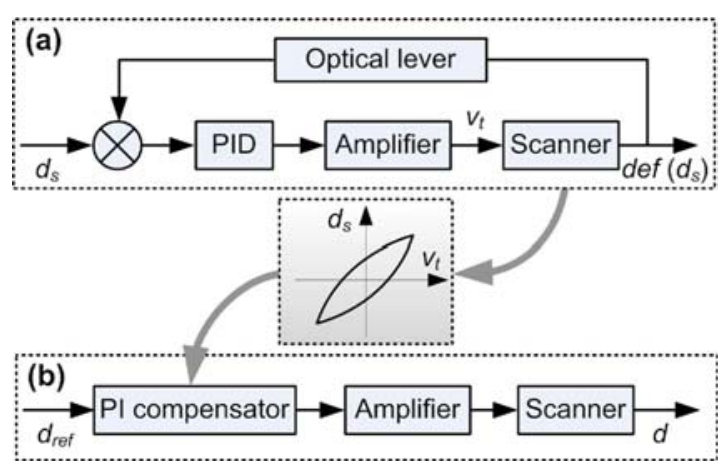

FIG. 2. (Color online) Method of hysteresis characterization and compensation with a PI compensator. (a) Bloc-setup of the compensator identification using the optical lever with the PID controller. (b) Feature use of the identified PI hysteresis model for hysteresis compensation.

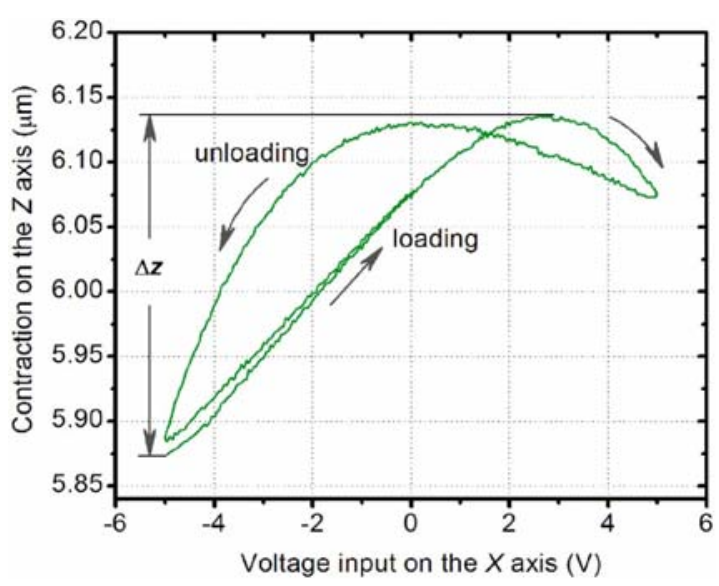

FIG. 3. (Color online) Contraction on the $Z$-axis when movement occurs over the full range of movement on the $X$-axis. It has been found that the maximum contraction $\Delta z$ can reach $262 \mathrm{~nm}$ considering the initial loading curve and $250 \mathrm{~nm}$ when taking no account of the initial loading effect.

as the hysteresis compensator for the piezoscanner, as shown in Fig. 2(b), in which $d_{\text {ref }}$ is a reference displacement input of the PI compensator and $d$ is a corresponding compensated displacement output of the piezoscanner.

However, hysteresis calibration on the $X$ - or the $Y$-axis is more complicated than calibration on the $Z$-axis due to contractions of the piezoscanner when movements occur on the $X$ - or the $Y$-axis. Figure 3 shows the contractions on the $Z$-axis obtained by scanning a very smooth plane on the $X$-axis using the tip I. The figure shows contractions of 262 and $250 \mathrm{~nm}$ taking and not taking account of the initial loading behavior, respectively. Software compensation of this type of contraction is feasible but complicated. Fortunately, when we calibrate the $X$-axis and $Y$-axis of the piezoscanner, another AFM cantilever in our two-tip nanomanipulation system, tip II in Fig. 1, can be used to eliminate the contractions by closed-loop control on the $Z$-axis with a constant reference position.

Figure 4(a) shows the hysteresis loop with five cycles on the $X$-axis of the piezoscanner as a sinusoidal input with a frequency of $0.5 \mathrm{~Hz}$ applied on the $X$-axis of nanopositioning stage, which shows the hysteresis on the $X$-axis reaches $28.14 \%(h \times 100 \% / H)$. The stable hysteresis loop was obtained after a few cycles of motion to eliminate the initial loading behavior of the piezoscanner. These data were acquired with a displacement of $12 \mu \mathrm{m}$ applied on the nanopositioning stage. The corresponding voltage input of the piezoscanner is $-5-4.79 \mathrm{~V}$, which indicates the actual displacement of the piezoelectric is more than $12 \mu \mathrm{m}$. Similar results were obtained on the calibration of the $Y$ - and the $Z$-axis, namely, 12 and $10 \mu \mathrm{m}$ with corresponding voltage inputs of $-5-4.87 \mathrm{~V}$ and $-5-4.96 \mathrm{~V}$, respectively. Once the hysteresis loop is ready, it is used to identify a corresponding PI model. To characterize the creep, we applied voltage steps from $-5 \mathrm{~V}$ on each axis of the piezoscanner and recorded the step responses with an interval of 0.02 and $100 \mathrm{~s}$ duration. The height of the voltage step on each axis was restricted within the voltage input range identified from the hysteresis characterization. Then the creep behavior of the piezoscanner was predicated by the logarithmic model. ${ }^{15}$ Open circles in 

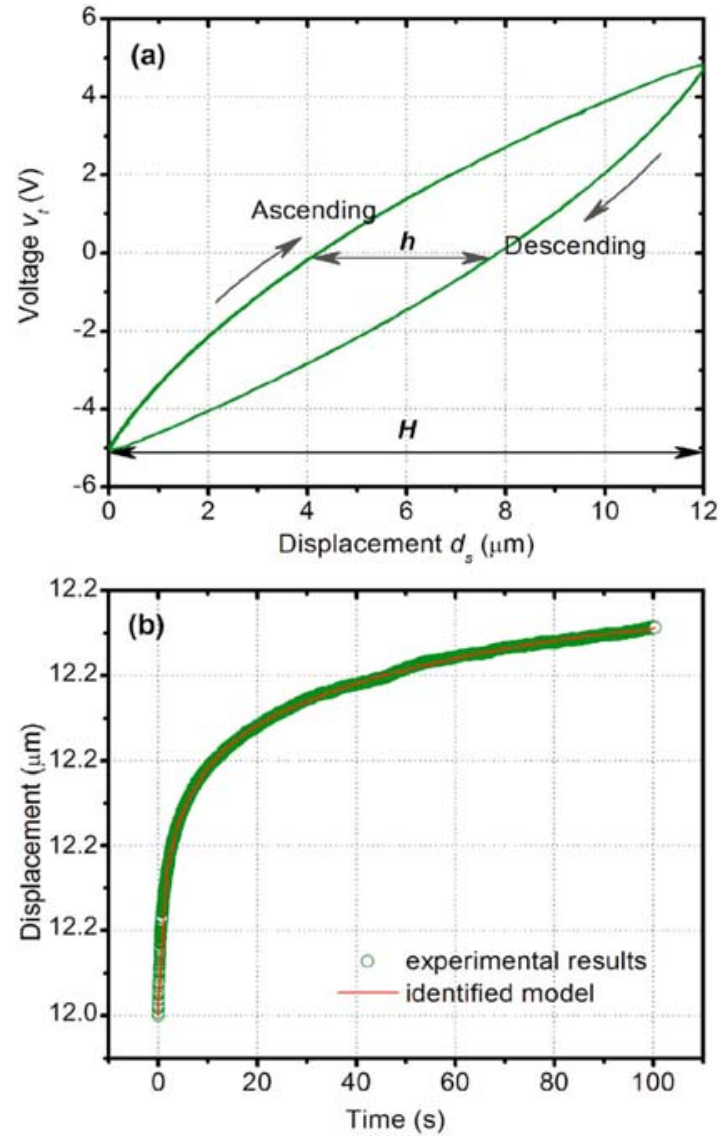

FIG. 4. (Color online) (a) Hysteresis loop with a sinusoidal input on the $X$-axis of the piezoscanner. A stable hysteresis loop was obtained after a few cycles of motion. These data were acquired with a displacement of $12 \mu \mathrm{m}$ applied on the $X$-axis of the nanopositioning stage. The corresponding voltage input of the piezoscanner was $-5-4.79 \mathrm{~V}$. (b) Identification of the creep behavior: experimental and simulation results.

Fig. 4(b) show a creep behavior with a step response from 0 to $12 \mu \mathrm{m}$ on the $X$-axis of the piezoscanner when a voltage step $-5-4.79 \mathrm{~V}$ was applied. The creep was clearly matched by the identified model shown by the red line.

Once the inverse PI model of the cycle pictured in Fig. 4(a) is identified, it is used as an inverse-based feedforward compensator for the piezoscanner. By combining the creep and the hysteresis, a software-based compensator would be able to handle precise actuation of the piezoscanner. Figure 5(a) shows an example of natural behavior on the $X$-axis when a sinusoidal voltage input with a frequency of $0.1 \mathrm{~Hz}$ and decreasing amplitude is applied. The creep effect is also shown to be very high due to the low input frequency. Figure 5(b) gives an experimental result with hysteresis and creep compensation, indicating that the hysteresis and the creep were well compressed, and the maximum error was reduced to $1.18 \%$ from $28.14 \%$ without compensation. It also reveals that the piezoscanner and the nanopositioning stage have an approximately equivalent motion scale. Similarly, after compensation, maximum errors on the $Y$-axis and the $Z$-axis are also reduced from $27.82 \%$ and $15.8 \%$ to $1.16 \%$ and $0.97 \%$, respectively.

In summary, we have presented a new method to achieve calibration of the piezoscanner that served as a nanopositioning device on our two-tip AFM-based nanomanipulation sys-
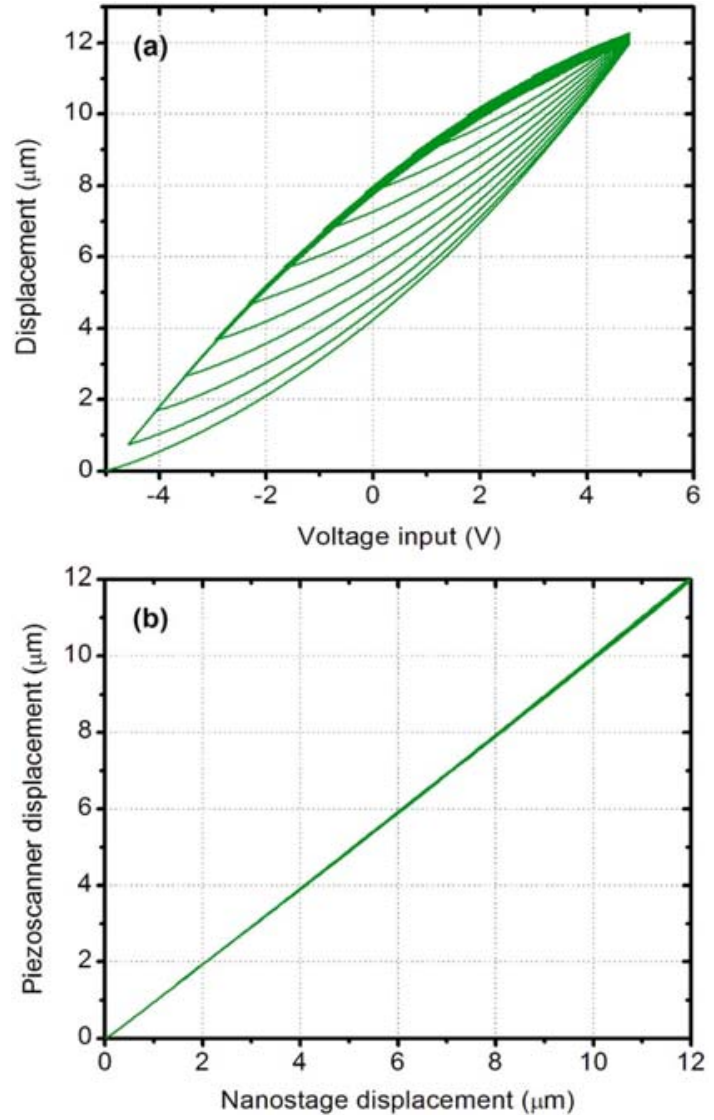

FIG. 5. (Color online) Example of the calibration results on the $X$-axis. (a) An ascending tracking result is recorded as voltage input vs displacement output of the piezoscanner on the $X$-axis without the hysteresis compensation. (b) An ascending tracking result on the $X$-axis with the reference input of piezoscanner vs nanopositioning stage displacement using hysteresis compensation

tem. This method uses the optical lever together with the cantilever as a displacement sensor to measure the displacement on the piezoscanner. A method such as this allows accurate characterization of the piezoscanner hysteresis and creep without any modification of the AFM setup or any additional calibration equipments.

${ }^{1}$ C. Newcomb and I. Filnn, Electron. Lett. 18, 442 (1982).

${ }^{2}$ K. Furutani, M. Urushibata, and N. Mohri, Nanotechnology 9, 93 (1998).

${ }^{3}$ L. Dupre, R. van Keer, and J. A. A. Melkebeek, J. Appl. Phys. 85, 4376 (1999).

${ }^{4}$ P. Ge and M. Jouaneh, IEEE Trans. Control Syst. Technol. 4, 209 (1996).

${ }^{5}$ R. Ben Mrad and H. Hu, IEEE/ASME Trans. Mechatron. 7, 479 (2002).

${ }^{6}$ R. C. Smith, J. Intell. Mater. Syst. Struct. 11, 62 (2000).

${ }^{7}$ M. Goldfarb and N. Celanovic, ASME J. Dyn. Syst., Meas., Control 119, 478 (1997).

${ }^{8}$ P. Krejci and K. Kuhnen, IEE Proc.: Control Theory Appl. 148, 185 (2001).

${ }^{9}$ M. Rakotondrabe, C. Clévy, and P. Lutz, IEEE Trans. Autom. Sci. Eng. (unpublished).

${ }^{10}$ B. Mokaberi and A. A. G. Requicha, IEEE. Trans. Autom. Sci. Eng. 5, 197 (2008).

${ }^{11}$ H. Jung, J. Y. Shim, and D. Gweon, Rev. Sci. Instrum. 71, 3436 (2000).

${ }^{12}$ D. Croft, G. Shed, and S. Devasia, ASME J. Dyn. Syst., Meas., Control 123,35 (2001).

${ }^{13}$ H. Xie, J. Vitard, S. Haliyo, S. Régnier, and M. Boukallel, Rev. Sci. Instrum. 79, 033708 (2008)

${ }^{14}$ H. Xie, J. Vitard, S. Haliyo, and S. Régnier, Rev. Sci. Instrum. 79, 096101 (2008).

${ }^{15}$ H. Jung and D. Gweon, Rev. Sci. Instrum. 71, 1896 (2000). 